\title{
Alcohol Use Among Patients With Cancer and Survivors in the United States, 2000-2017
}

Nina N. Sanford, MD; ; David J. Sher, MDa; Xiaohan Xu, MS,cc; Chul Ahn, PhDd,e; Anthony V. D'Amico, MD, PhDf; Ayal A. Aizer, MDf; and Brandon A. Mahal, MDs

\begin{abstract}
Background: Alcohol use is an established risk factor for several malignancies and is associated with adverse oncologic outcomes among individuals diagnosed with cancer. The prevalence and patterns of alcohol use among cancer survivors are poorly described. Methods: We used the National Health Interview Survey from 2000 to 2017 to examine alcohol drinking prevalence and patterns among adults reporting a cancer diagnosis. Multivariable logistic regression was used to define the association between demographic and socioeconomic variables and odds of self-reporting as a current drinker, exceeding moderate drinking limits, and engaging in binge drinking. The association between specific cancer type and odds of drinking were assessed. Results: Among 34,080 survey participants with a known cancer diagnosis, $56.5 \%$ self-reported as current drinkers, including $34.9 \%$ who exceeded moderate drinking limits and $21.0 \%$ who engaged in binge drinking. Younger age, smoking history, and more recent survey period were associated with higher odds of current, exceeding moderate, and binge drinking $(P<.001$ for all, except $P=.008$ for excess drinking). Similar associations persisted when the cohort was limited to 20,828 cancer survivors diagnosed $\geq 5$ years before survey administration. Diagnoses of melanoma and cervical, head and neck, and testicular cancers were associated with higher odds of binge drinking $(P<.05$ for all) compared with other cancer diagnoses. Conclusions: Most cancer survivors self-report as current alcohol drinkers, including a subset who seem to engage in excessive drinking behaviors. Given that alcohol intake has implications for cancer prevention and is a potentially modifiable risk factor for cancer-specific outcomes, the high prevalence of alcohol use among cancer survivors highlights the need for public health strategies aimed at the reduction of alcohol consumption.
\end{abstract}

J Natl Compr Canc Netw 2020;18(1):69-79 doi: 10.6004/jnccn.2019.7341

aDepartment of Radiation Oncology, and ${ }^{b}$ Division of Biostatistics, Department of Clinical Sciences, University of Texas Southwestern Medical Center, Dallas, Texas; ' Department of Statistical Science, Southern Methodist University,

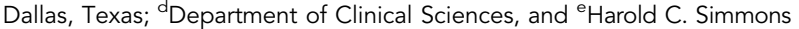
Comprehensive Cancer Center, University of Texas Southwestern Medical Center, Dallas, Texas; and fDepartment of Radiation Oncology, Dana-Farber/Brigham and Women's Hospital, and 9McGraw/Patterson Center for Population Sciences, Dana-Farber Cancer Institute, Boston, Massachusetts.

\section{Background}

Alcohol use has been identified as a risk factor for several malignancies, including cancers of the head and neck, esophagus, colorectum, liver, and breast. ${ }^{1-3}$ Epidemiologic studies have shown that higher alcohol consumption over longer time intervals increases an individual's risk for cancer, and some studies have suggested that even low levels of drinking seem to be associated with the development of certain cancer types. ${ }^{4,5}$ In addition to the link between alcohol use and cancer risk, emerging evidence suggests that alcohol is associated with worse oncologic outcomes among patients already diagnosed with cancer. ${ }^{6-10}$

As a result, the association between alcohol use and cancer has received increased recent attention. Using global estimates of patients with cancer and deaths from the WHO, one study estimated that $5.8 \%$ of cancer deaths were attributable to alcohol use in 2012, up from $3.6 \%$ in 2002. ${ }^{11}$ Major cancer organizations are explicitly focusing efforts to promote public health, research, and policy-related work directed toward obtaining a better understanding of the relationship between alcohol use and cancer, including the need to characterize drinking patterns among individuals diagnosed with cancer. ${ }^{12}$

No contemporary comprehensive investigations of drinking behaviors among adult cancer survivors have been conducted in the United States. Therefore, we used a contemporary nationwide database to characterize alcohol consumption behaviors among US cancer survivors.

\section{Methods}

Data Source

The National Health Interview Survey (NHIS) is a crosssectional household survey of noninstitutionalized civilian adults living in the United States that assesses a wide range of health status and utilization measures. ${ }^{13}$

See JNCCN.org for supplemental online content. 
First administered in 1957, the NHIS uses a multistage probability design to ensure broad geographic representation. Sample weights are provided for each individual, permitting inferences on national prevalence. Harmonized data were obtained through the Integrated Health Interview Series. ${ }^{14}$

\section{Population}

The study population included adults (aged $\geq 18$ years) sampled in 2000 through 2017 reporting a diagnosis of cancer. Participants were queried regarding their cancer history via the question: "Have you ever been told you had cancer?" Among participants who answered yes, the type of cancer was recorded. Those who reported a diagnosis of nonmelanoma skin cancer or skin cancer type unknown were excluded. Participants were also queried regarding current alcohol use and were asked to classify themselves as lifetime abstainers ( $<12$ drinks in life), former drinkers (no drinks in the past year), or current drinkers ( $\geq 1$ drinks in the past year). Individuals who were current drinkers were further asked the average number of drinks consumed on days they drank and how many days during the past year they had consumed $\geq 5$ drinks.

Additional sociodemographic variables included were age, sex, annual family income, race, ethnicity, marital status, smoking status, insurance status, highest education level, and self-reported health status.

\section{Quantification of Alcohol Intake}

Study participants were categorized as current drinkers, former drinkers, or lifetime abstainers (never drinkers). Among those who were current drinkers, exceeding moderate drinking level was defined as having $>1$ drink per day for women and $>2$ drinks per day for men, per CDC guidelines. ${ }^{15}$

Guidelines issued by the CDC and the National Institute on Alcohol Abuse and Alcoholism define binge drinking as the consumption of alcohol that raises the blood alcohol concentration (BAC) to $\geq 0.08 \%$, and specify that this pattern of drinking usually corresponds to $\geq 5$ drinks on a single occasional for men or $\geq 4$ drinks on a single occasion for women, generally within approximately 2 hours. ${ }^{16}$ For the purposes of this study, individuals including both men and women were classified as having participated in binge drinking if they had consumed $\geq 5$ drinks during at least 1 day over the past year. No information regarding BAC or time interval of drinking was available in our database.

The NHIS is overseen by the National Center for Health Statistics Research Ethics Review Board. Survey participants provided verbal informed consent. The University of Texas Southwestern Medical Center deemed the study exempt given the use of public deidentified data.

\section{Statistical Analysis}

\section{Baseline Characteristics}

Baseline characteristics, including demographic and socioeconomic variables, were reported for the entire cohort $(\mathrm{N}=34,080)$ and for the subgroup of cancer survivors diagnosed $\geq 5$ years before survey administration $(n=20,828)$.

\section{Alcohol Consumption Among Participants Diagnosed With Cancer}

The primary endpoint of interest in this study was current alcohol consumption. For each of the drinking levels-never drinker (vs current/former drinker), former drinker (vs never/current drinker), current drinker (vs former/never drinker), exceeding moderate drinking (yes vs no among current drinkers), and binge drinking (yes vs no among current drinkers)-multivariable logistic regression defined the adjusted odds ratio of meeting the criteria for the specified level of alcohol consumption (yes vs no) by demographic and socioeconomic variables, including age, sex, annual family income, race, ethnicity, marital status, smoking status, highest education level, insurance status, self-reported health status, and survey period. Time since cancer diagnosis was also included in the multivariable model to ascertain whether drinking patterns differed among participants recently diagnosed with cancer and potentially undergoing active treatment versus cancer survivors with a more remote oncologic history.

As a sensitivity analysis, we repeated the multivariable models, restricting the cohort to participants diagnosed with cancer $\geq 5$ years before survey administration. These models included the same variables noted earlier, with the exception of time since cancer diagnosis.

Finally, we restricted the cohort to cancer survivors aged $\leq 50$ years to assess trends in drinking patterns for younger cancer survivors, again using the same variables, with the exception of age.

\section{Alcohol Consumption by Cancer Type}

The odds of self-reporting current drinking, exceeding moderate drinking levels, and binge drinking by cancer type (ie, lung cancer vs all other cancers) were assessed using univariable logistic regression models. This analysis was conducted in a univariable fashion to ascertain which cancer sites included patient populations more likely to engage in drinking. Because the definitions of exceeding moderate drinking differed for women versus men, analyses regarding the odds of exceeding moderate drinking for sex-specific cancers (breast, cervix, uterus, prostate, and testicular) were restricted to only one sex. Similar to the earlier section on alcohol 
consumption among participants diagnosed with cancer, as a sensitivity analysis, patients diagnosed $<5$ years before survey administration were excluded from the analysis to mitigate the impact of the acute effects of diagnosis and treatment on alcohol consumption and to exclude those with poor disease prognoses.

Sample weighting stratified by year was used for all analyses to produce nationally representative estimates. Statistical testing was 2-sided, with $\alpha=0.05$. Analyses were performed using Stata/SE 15.1 (StataCorp LLP) or R version 3.0.2 (R Foundation for Statistical Computing).

\section{Results}

\section{Baseline Characteristics}

In 2000 through 2017, a total of 34,080 participants reported having a cancer history, including 20,828 reporting a cancer diagnosis $\geq 5$ years before survey administration. In the overall cohort, median age was 67 years (interquartile range [IQR], 55-76 years), with most participants being female (59.5\%) and white $(89.1 \%)$. More than half of participants were former $(37.8 \%)$ or current $(16.7 \%)$ smokers and reported their health status as good (32.8\%) or very good $(25.2 \%)$. The baseline characteristics of cancer survivors diagnosed $\geq 5$ years before survey administration were similar to those of the main cohort (Table 1).

\section{Alcohol Consumption by Clinical and \\ Sociodemographic Characteristics}

In the primary cohort, $56.5 \%$ of cancer survivors selfreported as current drinkers (Table 1). Factors associated with higher odds of current drinking were male sex, better self-reported health status, younger age, white race, non-Hispanic ethnicity, higher income, higher education, current or former smoking status, and later survey period $(P<.05$ for all, except for very good health status vs excellent, $P=.2$; Table 2 ).

Exceeding moderate drinking levels (classified as $>1$ drink for women and $>2$ drinks for men) ${ }^{15}$ was reported in $34.9 \%$ of current drinkers and was more common among female $(P<.001)$ and Alaska Native/American Indian study participants $(P=.05)$, and in those with annual family income $>\$ 100,000(P=.05)$ (Table 2).

Among cancer survivors, $21.0 \%$ of current drinkers also reported engaging in binge drinking. Variables associated with binge drinking included male sex, better health status, younger age, Alaska Native/American Indian race, higher income, former or current smoking status, and later survey period $(P<.001$ for all with the exception of $P=.04$ for Alaska Native/American Indian participants; Table 2).

Notably, alcohol drinking at all levels was more common among younger participants. For example, among those aged 18 to 34 years, $73.5 \%$ were current drinkers, including $35.5 \%$ who met the criteria for binge drinking. In contrast, $43.8 \%$ of cancer survivors aged $\geq 75$ years were current drinkers, including $2.6 \%$ who engaged in binge drinking (Figure 1).

Furthermore, drinking alcohol, and particularly engaging in binge drinking, was more prevalent among those who were current smokers. For example, $8.0 \%$ versus $23.6 \%$ of never versus current smokers reported binge drinking, respectively (Figure 2). The odds of current drinking, exceeding moderate drinking levels, and binge drinking all increased with later survey period $(P<.001$ for all except exceeding moderate drinking, $P=.008$; supplemental eFigure 1 , available with this article at JNCCN.org). There was no association between the interval since cancer diagnosis and the odds of alcohol consumption ( $P>.05$ for all). In contrast to levels of current drinking, cancer survivors who were women, were never smokers, had worse self-reported health status, and were older were more likely to self-report as former and never drinkers (Table 2).

Among 20,828 participants reporting a cancer diagnosis $\geq 5$ years before survey administration, $57.1 \%$ were current drinkers, including $35.1 \%$ exceeding moderate drinking limits and $20.1 \%$ engaging in binge drinking. Within this subgroup, similar variables were associated with the odds of alcohol consumption at all levels, including age at diagnosis and smoking status $(P<.001$ for all, except for age group 35-44 years vs 18-34 years) (supplemental eTable 1 ). Later survey period was associated with increased odds of current drinking $(P<.001)$ and binge drinking $(P<.001)$ but not of exceeding moderate drinking levels $(P=.84)$.

Among 6,313 cancer survivors aged $\leq 50$ years, the odds of binge drinking increased over time, but not the odds of current drinking or exceeding moderate drinking without binge drinking (supplemental eTable 2).

\section{Alcohol Consumption by Cancer Type}

Among participants diagnosed with cancer, those with cervical or testicular cancer were more likely to report drinking at all levels (Figure 3). In contrast, having a diagnosis of breast cancer was associated with lower odds of drinking at all levels. Among the 84 patients with male breast cancer, 48 (57.1\%) were current drinkers. Of those 84 patients who were currently drinking, 25 (52.1\%) exceeded moderate drinking levels and 11 (22.9\%) met the criteria for binge drinking (data not shown). Notably, patients with a history of head and neck cancer and/or melanoma, along with those diagnosed with cervical or testicular cancer, were also more likely to report binge drinking compared with those with other cancers. Similar trends were observed when the cohort 
Table 1. Baseline Patient Characteristics

\begin{tabular}{|c|c|c|c|c|}
\hline \multirow[b]{2}{*}{ Characteristic } & \multicolumn{2}{|c|}{$\begin{array}{l}\text { Cancer History (Diagnosis at Any Time, } \\
\qquad N=34,080)\end{array}$} & \multicolumn{2}{|c|}{$\begin{array}{c}\text { Cancer History (Diagnosis } \geq 5 \text { Years } \\
\text { Before Survey, } n=20,828 \text { ) }\end{array}$} \\
\hline & $\begin{array}{c}\text { Raw } \\
\text { Number }\end{array}$ & $\begin{array}{c}\text { Raw \%/ } \\
\text { Weighted \%a }\end{array}$ & $\begin{array}{c}\text { Raw } \\
\text { Number }\end{array}$ & $\begin{array}{c}\text { Raw \%/ } \\
\text { Weighted \% }\end{array}$ \\
\hline \multicolumn{5}{|l|}{ Drinking status } \\
\hline Never drinker & 7,069 & $20.7 / 19.2$ & 4,324 & $20.8 / 19.3$ \\
\hline Former drinker & 8,630 & $25.3 / 24.3$ & 5,121 & $24.6 / 23.6$ \\
\hline Current drinker & 18,381 & $53.9 / 56.5$ & 11,383 & $54.6 / 57.1$ \\
\hline Exceeding moderate drinking & 6,491 & $35.3 / 34.9$ & 4,025 & $35.3 / 35.1$ \\
\hline Binge drinking & 3,713 & $20.4 / 21.0$ & 2,193 & 19.5/20.1 \\
\hline Age, median (IQR), y & 67 & $(55-76)$ & 67 & $(56-77)$ \\
\hline \multicolumn{5}{|l|}{ Age } \\
\hline $18-34 y$ & 1,761 & $5.2 / 5.6$ & 900 & $4.3 / 4.8$ \\
\hline $35-44$ y & 2,328 & $6.8 / 7.5$ & 1,421 & $6.8 / 7.6$ \\
\hline $45-54$ y & 4,216 & $12.4 / 14.0$ & 2,484 & $11.9 / 13.5$ \\
\hline $55-64$ y & 6,933 & $20.3 / 21.7$ & 4,137 & $19.9 / 21.4$ \\
\hline $65-74 y$ & 8,750 & $25.7 / 24.6$ & 5,230 & $25.1 / 24.1$ \\
\hline$\geq 75$ y & 10,092 & $29.6 / 26.6$ & 6,656 & $32.0 / 28.6$ \\
\hline \multicolumn{5}{|l|}{ Sex } \\
\hline Male & 12,731 & $37.4 / 40.5$ & 7,059 & $33.9 / 36.6$ \\
\hline Female & 21,349 & $62.6 / 59.5$ & 13,769 & $66.1 / 63.4$ \\
\hline \multicolumn{5}{|l|}{ Annual family income ${ }^{b}$ (USD) } \\
\hline$\$ 0-\$ 34,999$ & 15,012 & $48.8 / 39.6$ & 9,215 & $48.7 / 39.6$ \\
\hline$\$ 35,000-\$ 74,999$ & 8,675 & $28.2 / 30.7$ & 5,319 & $28.1 / 30.8$ \\
\hline$\$ 75,000-\$ 99,999$ & 1,922 & $6.2 / 7.9$ & 1,190 & $6.3 / 7.8$ \\
\hline$\geq \$ 100,000$ & 3,127 & $10.2 / 14.3$ & 1,935 & $10.2 / 14.6$ \\
\hline Not ascertained/unknown & 2,622 & $6.7 / 7.4$ & 1,270 & $6.7 / 7.4$ \\
\hline \multicolumn{5}{|l|}{ Race $^{c}$} \\
\hline White & 29,288 & $86.1 / 89.1$ & 18,045 & $86.7 / 89.4$ \\
\hline Black & 3,565 & $10.5 / 7.8$ & 2,068 & $10.0 / 7.5$ \\
\hline Alaska Native/American Indian & 294 & $0.9 / 0.8$ & 165 & $0.8 / 0.6$ \\
\hline Asian & 751 & $2.2 / 2.1$ & 448 & $2.2 / 2.2$ \\
\hline Multiple/Unknown & 182 & $0.3 / 0.3$ & 102 & $0.5 / 0.4$ \\
\hline \multicolumn{5}{|l|}{ Ethnicity $^{c}$} \\
\hline Non-Spanish/Hispanic/Latino & 31,600 & $92.7 / 94.3$ & 19,458 & $93.4 / 94.8$ \\
\hline Spanish/Hispanic/Latino & 2,480 & $7.3 / 5.7$ & 1,369 & $6.6 / 5.2$ \\
\hline \multicolumn{5}{|l|}{ Marital status ${ }^{d}$} \\
\hline Not married & 18,675 & $54.8 / 41.4$ & 11,612 & $55.8 / 42.2$ \\
\hline Married & 15,346 & $45.2 / 58.6$ & 9,180 & $44.2 / 57.8$ \\
\hline \multicolumn{5}{|l|}{ Smoking status ${ }^{d}$} \\
\hline Never & 15,570 & $45.7 / 45.6$ & 9,526 & $46.3 / 46.4$ \\
\hline Former & 12,655 & $37.2 / 37.8$ & 7,542 & $36.2 / 36.3$ \\
\hline Current & 5,822 & $17.1 / 16.7$ & 3,645 & $17.5 / 17.4$ \\
\hline \multicolumn{5}{|l|}{ Insurance status ${ }^{d}$} \\
\hline Insurance & 32,125 & $94.4 / 94.3$ & 19,520 & $93.8 / 93.7$ \\
\hline No insurance & 1,918 & $5.6 / 5.7$ & 1,287 & $6.2 / 6.3$ \\
\hline
\end{tabular}


Table 1. Baseline Patient Characteristics (cont.)

\begin{tabular}{|c|c|c|c|c|}
\hline \multirow[b]{2}{*}{ Characteristic } & \multicolumn{2}{|c|}{$\begin{array}{l}\text { Cancer History (Diagnosis at Any Time, } \\
\qquad N=34,080)\end{array}$} & \multicolumn{2}{|c|}{$\begin{array}{c}\text { Cancer History (Diagnosis } \geq 5 \text { Years } \\
\text { Before Survey, } n=20,828 \text { ) }\end{array}$} \\
\hline & $\begin{array}{c}\text { Raw } \\
\text { Number }\end{array}$ & $\begin{array}{c}\text { Raw \%/ } \\
\text { Weighted \%a }\end{array}$ & $\begin{array}{l}\text { Raw } \\
\text { Number }\end{array}$ & $\begin{array}{l}\text { Raw \%/ } \\
\text { Weighted \% }\end{array}$ \\
\hline \multicolumn{5}{|l|}{ Education } \\
\hline None-grade 4 & 462 & $1.4 / 1.1$ & 246 & $1.2 / 1.0$ \\
\hline Grade 5-12 & 15,372 & $45.1 / 44.0$ & 9,346 & $44.9 / 43.8$ \\
\hline $1-4$ y college & 14,698 & $43.1 / 43.9$ & 9,048 & $43.4 / 44.1$ \\
\hline$\geq 5$ y college & 3,378 & $9.9 / 10.5$ & 2,100 & $10.1 / 10.6$ \\
\hline Unknown & 170 & $0.5 / 0.5$ & 88 & $0.4 / 0.5$ \\
\hline \multicolumn{5}{|l|}{ Self-reported health status ${ }^{d}$} \\
\hline Excellent & 4,075 & $12.0 / 12.8$ & 2,742 & $13.2 / 14.2$ \\
\hline Very good & 8,328 & $24.5 / 25.2$ & 5,349 & $25.7 / 26.6$ \\
\hline Good & 11,229 & $33.0 / 32.8$ & 6,883 & $33.1 / 32.4$ \\
\hline Fair & 7,204 & $21.2 / 20.2$ & 4,148 & 19.9/19.1 \\
\hline Poor & 3,197 & $9.4 / 9.0$ & 1,685 & $8.1 / 7.7$ \\
\hline \multicolumn{5}{|l|}{ Survey period } \\
\hline $2000-2004$ & 8,313 & $24.4 / 22.0$ & 4,803 & $23.1 / 20.7$ \\
\hline $2005-2009$ & 7,516 & $22.1 / 26.5$ & 4,560 & $21.9 / 26.4$ \\
\hline 2010-2014 & 10,976 & $32.2 / 30.6$ & 6,831 & $32.8 / 31.0$ \\
\hline $2015-2017$ & 7,275 & $21.4 / 21.0$ & 4,634 & $22.3 / 21.9$ \\
\hline \multicolumn{5}{|l|}{ Interval since diagnosis } \\
\hline $0-5 y$ & 13,446 & $39.5 / 40.3$ & $\mathrm{~N} / \mathrm{A}$ & \\
\hline $6-10 y$ & 6,883 & $20.2 / 20.5$ & $\mathrm{~N} / \mathrm{A}$ & \\
\hline $11-15 y$ & 3,663 & $10.8 / 10.6$ & $\mathrm{~N} / \mathrm{A}$ & \\
\hline$\geq 16 y$ & 10,088 & $29.6 / 28.6$ & $\mathrm{~N} / \mathrm{A}$ & \\
\hline \multicolumn{5}{|l|}{ Cancer type } \\
\hline Breast & 8,418 & $24.7 / 23.2$ & 5,394 & $25.9 / 24.9$ \\
\hline Prostate & 5,182 & $15.2 / 15.9$ & 2,902 & $13.9 / 14.8$ \\
\hline Cervical & 3,308 & $9.7 / 9.6$ & 2,522 & $12.1 / 12.0$ \\
\hline Colorectal & 3,283 & $9.6 / 9.9$ & 1,835 & $8.8 / 8.9$ \\
\hline Melanoma & 3,100 & $9.1 / 8.7$ & 1,790 & $8.6 / 8.7$ \\
\hline Uterine & 2,298 & $6.7 / 6.2$ & 1,659 & $8.0 / 7.8$ \\
\hline Lung & 1,454 & $4.3 / 4.1$ & 546 & $2.6 / 2.4$ \\
\hline Bladder & 1,157 & $3.4 / 3.8$ & 614 & $2.9 / 3.6$ \\
\hline Head and neck & 794 & $2.3 / 2.3$ & 454 & $2.2 / 2.0$ \\
\hline Central nervous system & 347 & $1.0 / 0.9$ & 181 & $0.9 / 1.0$ \\
\hline Testicular & 338 & $1.0 / 1.0$ & 253 & $1.2 / 1.1$ \\
\hline Liver & 320 & $0.9 / 1.2$ & 98 & $0.5 / 0.6$ \\
\hline
\end{tabular}

Abbreviations: IQR, interquartile range; NHIS, National Health Information Survey.

aSample weighting stratified by year was used for all analyses to produce nationally representative estimates. Percentages may not add up to $100 \%$ due to rounding. ${ }^{b}$ Raw column numbers for income levels do not add up to the total participant number. Between 2000-2006, survey participants were given an income option of " $\geq \$ 75,000$ " and " $\geq \$ 20,000$." The 2,722 participants who chose one of these options are not included in the table because of ambiguous income levels.

cRace and ethnicity were self-reported as captured by the NHIS. Participants were asked whether they identified with one or more of the following racial groups: white, black/African American, Alaskan Native or American Indian, Chinese, Filipino, Asian Indian, or other Asian. Those reporting Chinese, Filipino, Asian Indian, or other Asian race were grouped as "Asian." Participants with multiple races or the primary race not releasable were grouped as "Unknown/Multiple." Participants were also asked whether they identified with one or more of the following ethnicities: not Hispanic/Spanish origin, Mexican, Mexican American, Puerto Rican, Cuban/Cuban American, Dominican (Republic), Central or South American, other Latin American (type not specific), other Spanish, or multiple Hispanic. Those reporting any of these ethnicities with the exception of not Hispanic/Spanish origin were categorized as "Spanish-Hispanic-Latino."

${ }^{d}$ Columns for marital status, smoking status, insurance, and self-reported health status do not add up to the total participant number because of 59, 33, 37, and 47 participants with unknown marital status, smoking status, insurance, and self-reported health status, respectively. 
Table 2. Multivariable Analysis of Participants $(\mathrm{N}=34,080)$

\section{Current Drinker}

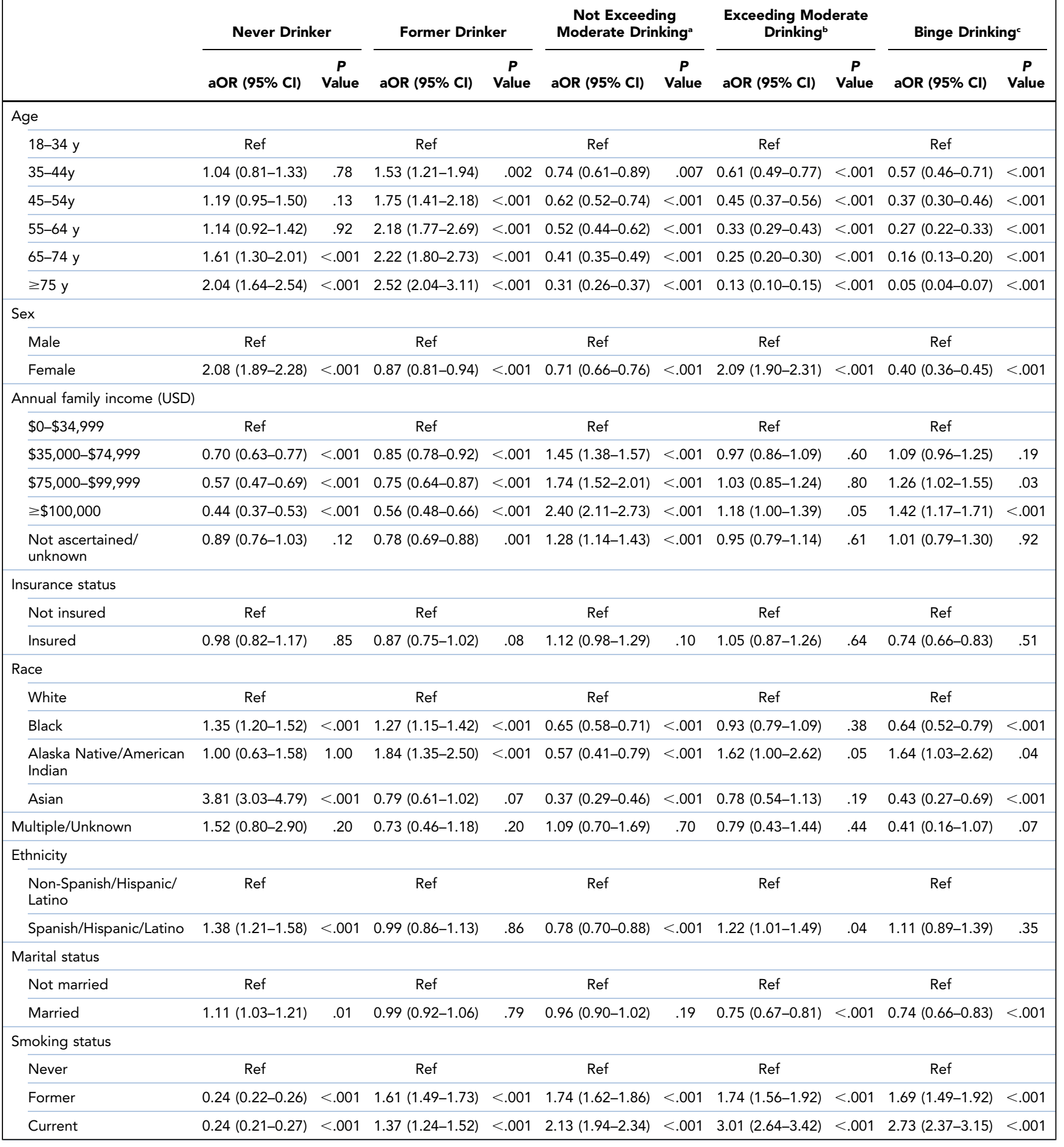


Table 2. Multivariable Analysis of Participants $(N=34,080)$ (cont.)

Current Drinker

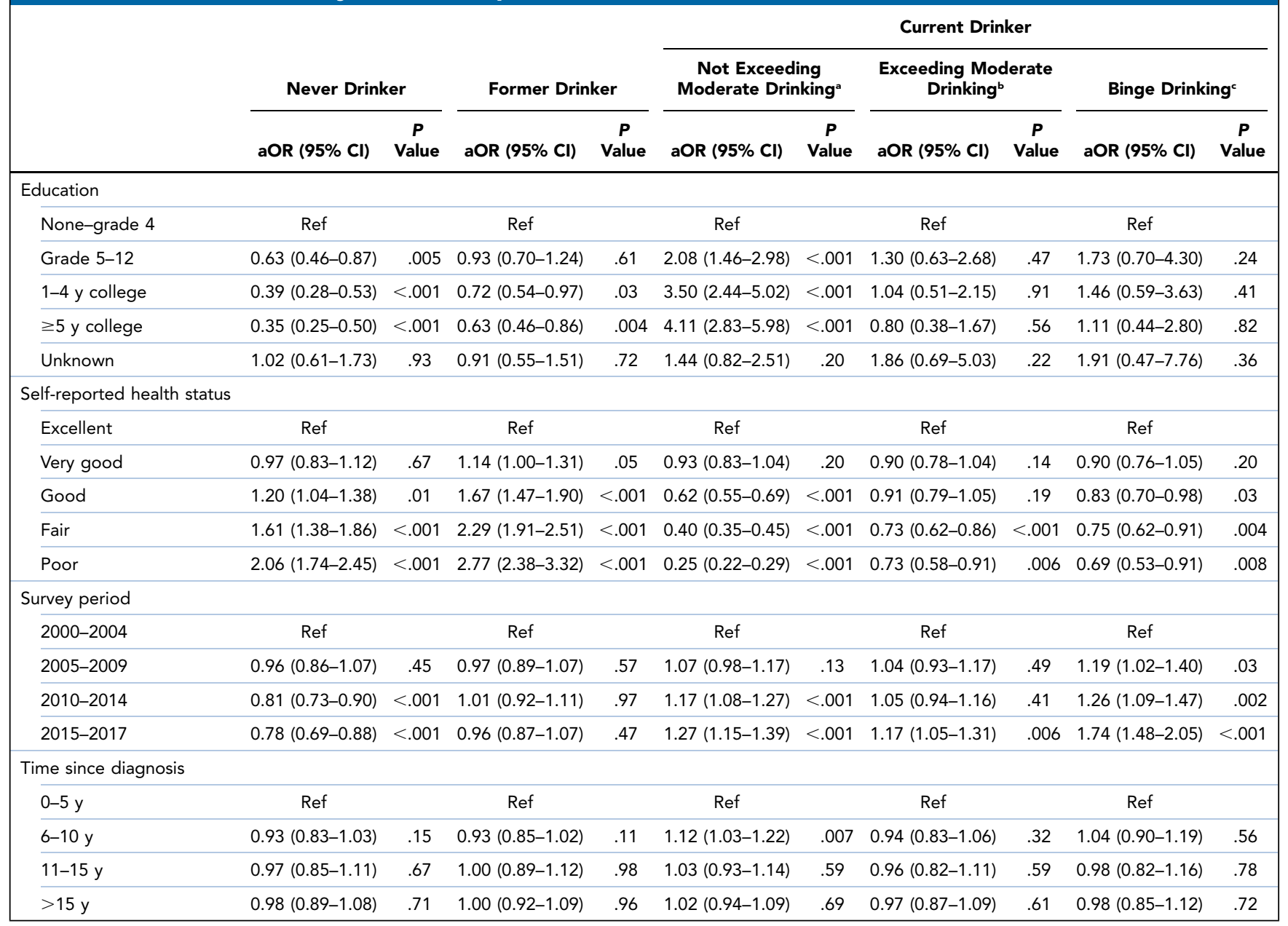

Abbreviation: aOR, adjusted odds ratio.

${ }^{a}$ Current drinking without meeting criteria for exceeding moderate drinking or binge drinking.

bDefined as $>1$ drink/day for women and $>2$ drinks/day for men, and includes those who engage in binge drinking

'Defined as $\geq 5$ drinks/day during at least 1 day over the past year.

was restricted to cancer survivors diagnosed $\geq 5$ years before survey administration (supplemental eTable 3).

\section{Discussion}

In this comprehensive population-based study of alcohol consumption among adult cancer survivors, most participants with a history of cancer $(56.5 \%)$ self-reported as current drinkers, including 34.9\% who exceeded moderate drinking limits and $21.0 \%$ who engaged in binge drinking. Younger age, current or former smoking status, and later survey period were associated with greater odds of currently drinking alcohol at all levels. The proportion of participants who self-reported alcohol consumption did not seem to change with an increasing interval since cancer diagnosis. Those with a history of melanoma and/or cervical, head and neck, and/or testicular cancer were more likely to engage in binge drinking, likely a reflection of the predominant demographic characteristics-particularly younger age-associated with these cancer diagnoses, rather than an intrinsic association between cancer type and alcohol use.

This study shows a high prevalence of alcohol use among cancer survivors and as such has important public health implications. First, mounting data suggest that alcohol use may be a risk factor for cancer and that continued alcohol use after a cancer diagnosis may be associated with worse oncologic outcomes. ${ }^{4,5,7,8}$ Given that alcohol intake is a potentially modifiable risk factor, policies and guidelines should be initiated to encourage the reduction of alcohol drinking among cancer survivors. By highlighting that most cancer survivors engage in drinking and identifying groups at higher risk for excessive drinking behaviors, our findings could serve as an impetus for a greater focus on alcohol screening and reduction as a part of cancer care. Second, this study shows a gradual 


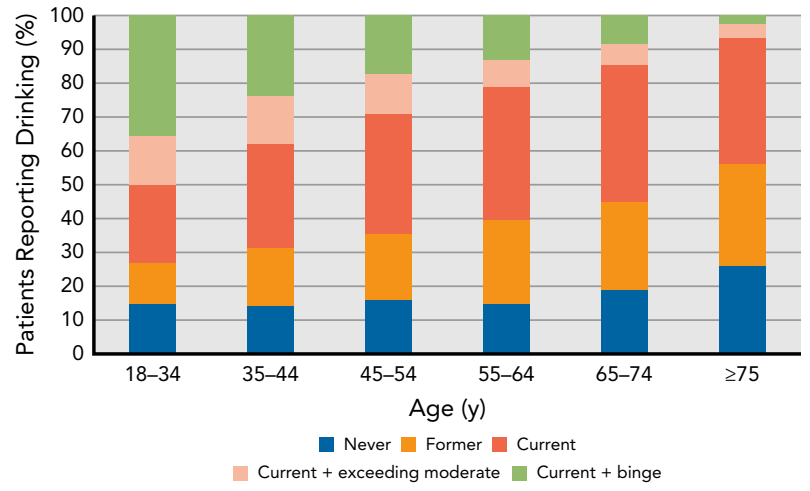

Figure 1. Proportion of study participants self-reporting as never, former, and current drinkers (including those who drink without exceeding moderate drinking limits, those who exceed moderate drinking limits, and those who engage in binge drinking) stratified by age category.

but persistent trend in increasing alcohol intake among cancer survivors over the past decade, particularly in binge drinking. Given the prognostic role of alcohol in cancer-specific outcomes, the factors contributing to this trend warrant further investigation.

The observed higher likelihood of alcohol intake at all levels among patients who are current smokers merits further discussion. Continued smoking after a cancer diagnosis has been associated with a wide range of adverse outcomes, including increased risks of cancer recurrence, more severe treatment-related toxicity, and development of a second primary cancer. ${ }^{17}$ Given the synergism between alcohol intake and smoking on the pathogenesis of several cancer subtypes, ${ }^{18,19}$ continued alcohol intake, particularly in large quantities, may pose a particularly negative health risk for current smokers. Notably, these risks are not limited to cancer-specific outcomes but also include stroke and cardiovascular disease, which smoking and heavy alcohol use, along with prior cancer treatments such as systemic therapy and radiotherapy, have been shown to negatively impact. ${ }^{20-22}$ Therefore, smokers represent a high-risk population that should also be appropriately screened and counseled regarding alcohol use. Furthermore, because drinking has been shown to represent a conduit for smoking ${ }^{23-26}$ (ie, individuals tend to smoke more after consuming alcohol), reducing the incidence of drinking could also potentially lower smoking rates, particularly in instances of co-use.

Notably, among cancer survivors, better selfreported health status was associated with higher odds of current drinking at all levels and, conversely, lower odds of being a former drinker. It is possible that individuals with a diagnosis of cancer who self-report poor health status may be those with persistent or recurrent disease who are undergoing active treatment and who have been advised not to drink or do not feel well enough to consume

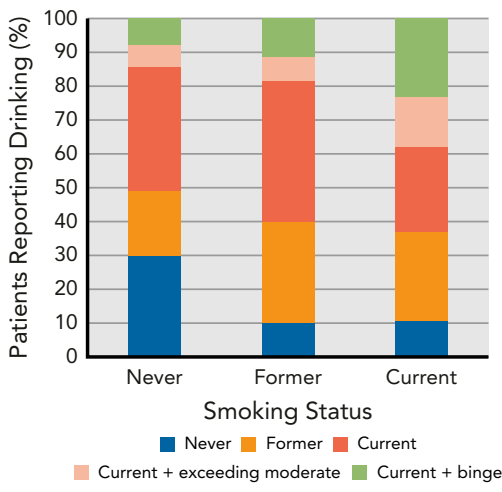

Figure 2. Proportion of study participants self-reporting as never, former, and current drinkers (including those who drink without exceeding moderate drinking limits, those who exceed moderate drinking limits, and those who engage in binge drinking) stratified by smoking status.

alcohol. Another interpretation of these findings is that alcohol could improve overall health among cancer survivors, which has been suggested in a recent study. ${ }^{27}$ It is important to note, however, that the NHIS database is participant-reported and that self-reported health statuses were not verified by objective measures.

Although white race was associated with higher odds of any current drinking among cancer survivors, Alaska Natives/American Indians had the highest odds of exceeding moderate drinking levels and engaging in binge drinking. Research on the general population has also reported higher rates of alcohol abuse among the Alaska Native/American Indian population, although practices vary greatly from tribe to tribe. ${ }^{28}$ Compared with other racial backgrounds, Alaska Natives/American Indians seem to be at higher risk for alcohol-related consequences, with one study showing a 5.6 times higher age-adjusted alcohol-related death rate. ${ }^{29}$ The causes of this disparity are likely multifactorial, with notable contributions from systemic factors partly rooted in structural racism, including limited healthcare resources and poor access to housing, education, and nutrition, all of which have been shown to affect health risk and outcomes. ${ }^{30,31}$ As such, the higher rates of excessive drinking behaviors among Alaska Native/American Indian cancer survivors should prompt the establishment of policies and guidelines to improve access to a wide range of resources affecting health outcomes among this population, including housing and education. Oncology providers working in areas with high Alaska Native/American Indian populations should be given appropriate time and resources to engage in culturally sensitive discussions regarding alcohol use.

This study has several notable limitations. First, the thresholds for moderate drinking and binge drinking used herein were those issued for the general population. The risks associated with varying levels of alcohol use 
A

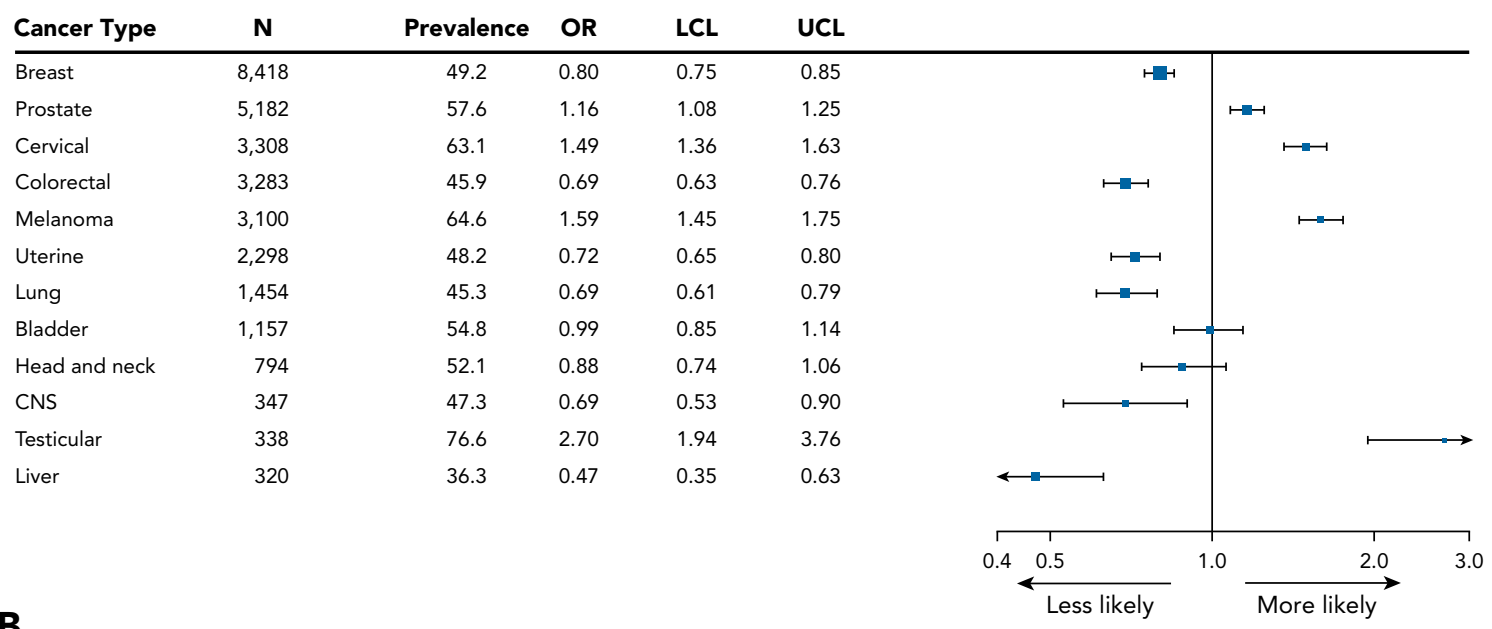

Cancer Type

Prevalence $\quad$ OR $\quad$ LCL $\quad$ UCL

\begin{tabular}{lllll}
\hline Breast & 37.4 & 0.67 & 0.60 & 0.74 \\
Prostate & 18.0 & 0.56 & 0.49 & 0.65 \\
Cervical & 61.2 & 2.29 & 2.02 & 2.60 \\
Colorectal & 28.8 & 0.80 & 0.68 & 0.93 \\
Melanoma & 33.2 & 0.95 & 0.84 & 1.08 \\
Uterine & 43.6 & 0.89 & 0.76 & 1.04 \\
Lung & 31.9 & 0.84 & 0.68 & 1.04 \\
Bladder & 22.5 & 0.58 & 0.45 & 0.73 \\
Head and neck & 35.6 & 0.91 & 0.71 & 1.17 \\
CNS & 36.6 & 1.15 & 0.78 & 1.71 \\
Testicular & 31.1 & 1.58 & 1.14 & 2.19 \\
Liver & 39.8 & 0.94 & 0.59 & 1.49
\end{tabular}

C

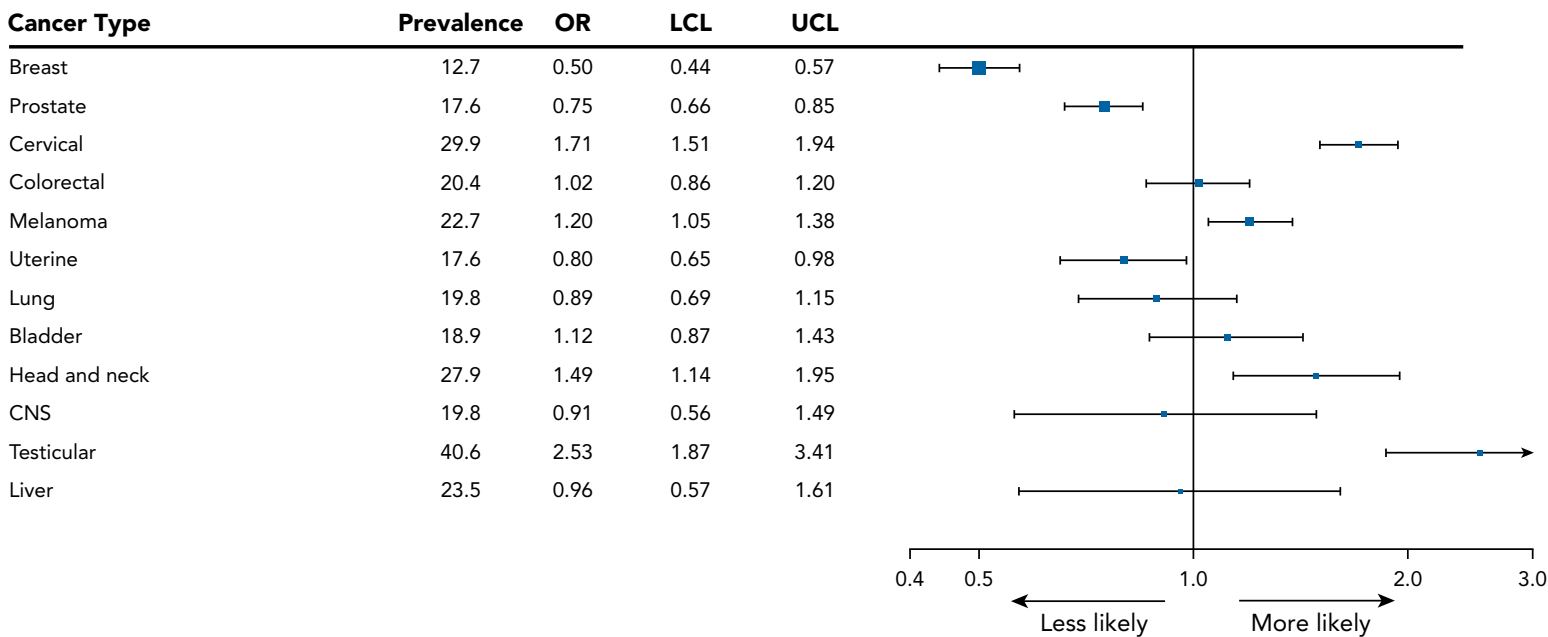

Figure 3. Forest plot for odds and associated $95 \% \mathrm{Cl}$ of self-reporting (A) current drinking, (B) exceeding moderate drinking, and (C) binge drinking. Only cancer types with $>300$ participants are shown. Each cancer is compared with all other cancer types.

Abbreviations: CNS, central nervous system; LCL, lower confidence limit; $\mathrm{OR}$, odds ratio; $\mathrm{UCL}$, upper confidence limit. 
may differ depending on underlying conditions, but no such guidelines have been created specifically for patients with cancer. Second, the CDC guidelines used in this study specified binge drinking as achieving a BAC of $0.08 \%$ and estimated this level to require 5 drinks in men and 4 drinks in women consumed over the course of $\leq 2$ hours. Information regarding the duration of alcohol intake was not available in the database, and therefore individuals reporting drinking $\geq 5$ drinks on one occasion may have done so over longer periods, and thus would not have reached the biological threshold for binge drinking. Third, alcohol intake in this survey was selfreported and not verified by any other sources such as family members. However, earlier research has suggested that individuals tend to underreport alcohol use, and that difference between reported and actual alcohol consumption is greatest in heavy drinkers. ${ }^{32,33}$ Therefore, the true prevalence and quantity of alcohol intake, particularly for binge drinking, may be higher than indicated by the NHIS survey. Fourth, study participants could report up to 3 cancer types, and information regarding the number of cancer types that each participant reported was not available. Therefore, the current analysis on the association between cancer type and current drinking likely included patients whose drinking status may have been affected by more than one cancer diagnosis. However, given that there were a total of 34,080 cancer survivors and 36,813 cancer types reported, the number of patients with more than one diagnosis was likely small.

\section{Conclusions}

Study findings show that most cancer survivors selfreport as current drinkers, including a subset who seem to engage in excessive drinking behaviors. By reporting the demographic and socioeconomic variables associated with alcohol intake, our work begins to identify subgroups toward whom alcohol-based interventions could be targeted, and could serve as a benchmark for assessing changes in drinking behavior in the population of patients with cancer. Nevertheless, given that this represents the first study to assess drinking patterns in cancer survivors, further investigation is needed, including large-scale systems-based research on alcohol use in cancer survivors. For the time being, because oncologists have a responsibility to promote the overall health and well-being of their patients, efforts should be undertaken to accurately assess alcohol intake among cancer survivors and to inform these individuals of the potential harms associated with continued drinking.

Submitted January 22, 2019; accepted for publication July 22, 2019

Author contributions: Study concept and design: Sanford, Mahal. Data acquisition: Sanford, Mahal. Data analysis and interpretation: Sanford, Mahal. Methodology: All authors. Manuscript writing: Sanford, Mahal. Manuscript review and editing: Sher, Xu, Ahn, D'Amico, Aizer.

Disclosures: The authors have disclosed that they have not received any financial considerations from any person or organization to support the preparation, analysis, results, or discussion of this article.

Correspondence: Nina N. Sanford, MD, Department of Radiation Oncology, University of Texas Southwestern Medical Center, 2280 Inwood Road, Dallas, TX 75390-9303. Email: Nina.Sanford@UTSouthwestern.edu

\section{References}

1. van't Veer P, Kampman E. Food, Nutrition, Physical Activity, and the Prevention of Cancer: A Global Perspective. Washington, DC: World Cancer Research Fund/American Institute for Cancer Research; 2007.

2. World Cancer Research Fund/American Institute for Cancer Research. Continuous Update Project Expert Report 2018. Diet, nutrition, physical activity and liver cancer. Available at: dietandcancerreport.org Accessed October 20, 2019.

3. IARC Working Group on the Evaluation of Carcinogenic Risks to Humans. Personal habits and indoor combustions. Volume $100 \mathrm{E}$. A review of human carcinogens. IARC Monogr Eval Carcinog Risks Hum 2012;100: $1-538$.

4. Scoccianti C, Cecchini M, Anderson AS, et al. European Code Against Cancer, 4th edition: alcohol drinking and cancer. Cancer Epidemiol 2016; 45:181-188

5. Cao Y, Willett WC, Rimm EB, et al. Light to moderate intake of alcohol, drinking patterns, and risk of cancer: results from two prospective US cohort studies. BMJ 2015;351:h4238.

6. Do KA, Johnson MM, Doherty DA, et al. Second primary tumors in patients with upper aerodigestive tract cancers: joint effects of smoking and alcohol (United States). Cancer Causes Control 2003;14:131-138.

7. Holm M, Olsen A, Christensen J, et al. Pre-diagnostic alcohol consumption and breast cancer recurrence and mortality: results from a prospective cohort with a wide range of variation in alcohol intake. Int $\mathrm{J}$ Cancer 2013;132:686-694.

8. Kwan $\mathrm{ML}$, Kushi $\mathrm{LH}$, Weltzien $\mathrm{E}$, et al. Alcohol consumption and breast cancer recurrence and survival among women with early-stage breast cancer: the life after cancer epidemiology study. J Clin Oncol 2010;28: 4410-4416.
9. Li Y, Mao Y, Zhang Y, et al. Alcohol drinking and upper aerodigestive tract cancer mortality: a systematic review and meta-analysis. Oral Oncol 2014;50:269-275.

10. Schwedhelm C, Boeing H, Hoffmann G, et al. Effect of diet on mortality and cancer recurrence among cancer survivors: a systematic review and meta-analysis of cohort studies. Nutr Rev 2016;74:737-748.

11. Praud D, Rota M, Rehm J, et al. Cancer incidence and mortality attributable to alcohol consumption. Int J Cancer 2016;138:1380-1387.

12. LoConte NK, Brewster AM, Kaur JS, et al. Alcohol and cancer: a statement of the American Society of Clinical Oncology. J Clin Oncol 2018;36:83-93.

13. Centers for Disease Control and Prevention. National Health Interview Survey: methods. Available at: http://www.cdc.gov/nchs/nhis/methods.htm. Accessed July 1, 2018

14. Blewett LA, Rivera Drew JA, Griffin R, et al. IPUMS Health Surveys: National Health Interview Survey, Version 6.3. Available at: https:// ipums.org/projects/ipums-health-surveys/d070.v6.3. Accessed July 30, 2019.

15. Centers for Disease Control and Prevention. Fact sheets - alcohol use and your health. Available at: https://www.cdc.gov/alcohol/fact-sheets/ alcohol-use.htm. Accessed July 30, 2019

16. Centers for Disease Control and Prevention. Alcohol and public health frequently asked questions. Available at: https://www.cdc.gov/alcohol/ faqs.htm. Accessed July 30, 2019

17. Chen AM, Chen LM, Vaughan A, et al. Tobacco smoking during radiation therapy for head-and-neck cancer is associated with unfavorable outcome. Int J Radiat Oncol Biol Phys 2011;79:414-419. 
18. Hashibe M, Brennan P, Chuang SC, et al. Interaction between tobacco and alcohol use and the risk of head and neck cancer: pooled analysis in the International Head and Neck Cancer Epidemiology Consortium. Cancer Epidemiol Biomarkers Prev 2009;18:541-550.

19. Prabhu A, Obi KO, Rubenstein JH. The synergistic effects of alcohol and tobacco consumption on the risk of esophageal squamous cell carcinoma: a meta-analysis. Am J Gastroenterol 2014;109:822-827.

20. Centers for Disease Control and Prevention. How Tobacco Smoke Causes Disease: The Biology and Behavioral Basis for Smoking-Attributable Disease. A Report of the Surgeon General. Atlanta, GA: Centers for Disease Control and Prevention; 2010.

21. Larsson SC, Wallin A, Wolk A, et al. Differing association of alcohol consumption with different stroke types: a systematic review and metaanalysis. BMC Med 2016;14:178.

22. Whitman IR, Agarwal V, Nah G, et al. Alcohol abuse and cardiac disease. J Am Coll Cardiol 2017:69:13-24.

23. Gubner NR, Thrul J, Kelly OA, et al. Young adults report increased pleasure from smoking cigarettes when drinking alcohol but not when using marijuana. Addict Res Theory 2018;26:71-76.

24. Bobo JK, Husten C. Sociocultural influences on smoking and drinking. Alcohol Res Health 2000;24:225-232.

25. Falk DE, Yi HY, Hiller-Sturmhöfel S. An epidemiologic analysis of cooccurring alcohol and tobacco use and disorders: findings from the National Epidemiologic Survey on Alcohol and Related Conditions. Alcohol Res Health 2006;29:162-171.
26. Weitzman ER, Chen YY. The co-occurrence of smoking and drinking among young adults in college: national survey results from the United States. Drug Alcohol Depend 2005;80:377-386.

27. Downer MK, Kenfield SA, Stampfer MJ, et al. Alcohol intake and risk of lethal prostate cancer in the Health Professionals Follow-Up Study. J Clin Oncol 2019:37:1499-1511.

28. Whitesell NR, Beals J, Crow CB, et al. Epidemiology and etiology of substance use among American Indians and Alaska Natives: risk, protection, and implications for prevention. Am J Drug Alcohol Abuse 2012;38:376-382.

29. Beauvais F. American Indians and alcohol. Alcohol Health Res World 1998;22:253-259.

30. Bailey ZD, Krieger N, Agénor M, et al. Structural racism and health inequities in the USA: evidence and interventions. Lancet 2017;389: 1453-1463.

31. Hardeman RR, Murphy KA, Karbeah J, et al. Naming institutionalized racism in the public health literature: a systematic literature review. Public Health Rep 2018;133:240-249.

32. Northcote J, Livingston M. Accuracy of self-reported drinking: observational verification of "last occasion" drink estimates of young adults. Alcohol Alcohol 2011;46:709-713.

33. Boniface $\mathrm{S}$, Kneale J, Shelton N. Drinking pattern is more strongly associated with under-reporting of alcohol consumption than sociodemographic factors: evidence from a mixed-methods study. BMC Public Health 2014;14:1297. 
Supplemental online content for:

\section{Alcohol Use Among Patients With Cancer and Survivors in the United States, 2000-2017}

Nina N. Sanford, MD; David J. Sher, MD; Xiaohan Xu, MS; Chul Ahn, PhD; Anthony V. D'Amico, MD, PhD;

Ayal A. Aizer, MD; and Brandon A. Mahal, MD

J Natl Compr Canc Netw 2020;18(1):69-79

eFigure 1: Adjusted ORs and Absolute Percents for Study Participants Self-Reporting Drinking eTable 1: Multivariable Analysis of Participants Reporting a Cancer Diagnosis > 5 Years Before Survey Administration eTable 2: Multivariable Analysis by Era of Survey Among Participants Aged $\leq 50$ Years

eTable 3: Multivariable Analysis by Cancer Type of Participants Reporting a Cancer Diagnosis $\geq 5$ Years Before Survey Administration 
A

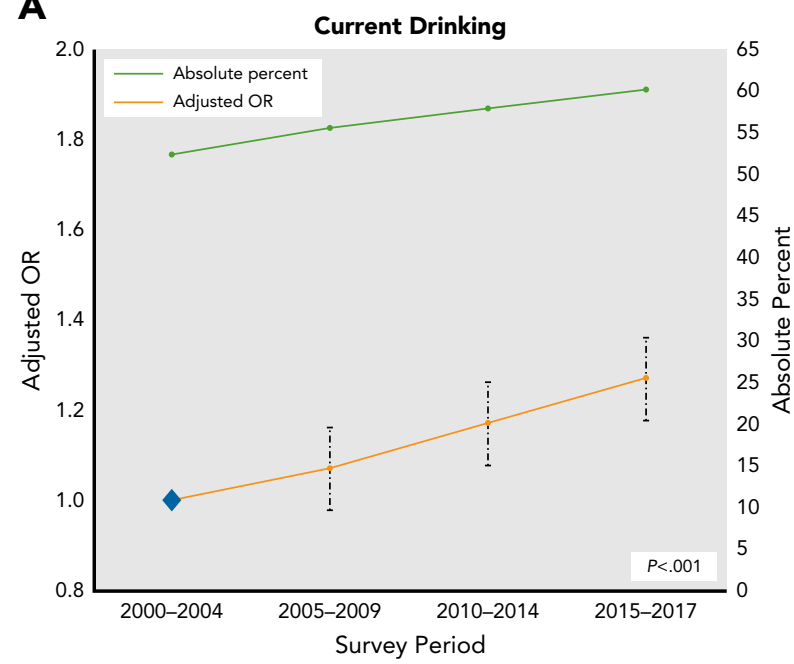

C

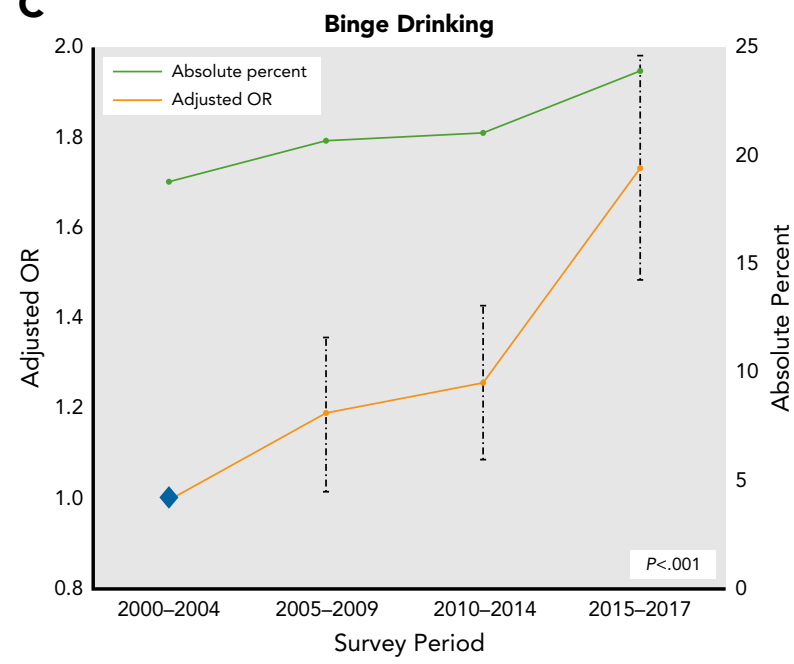

B

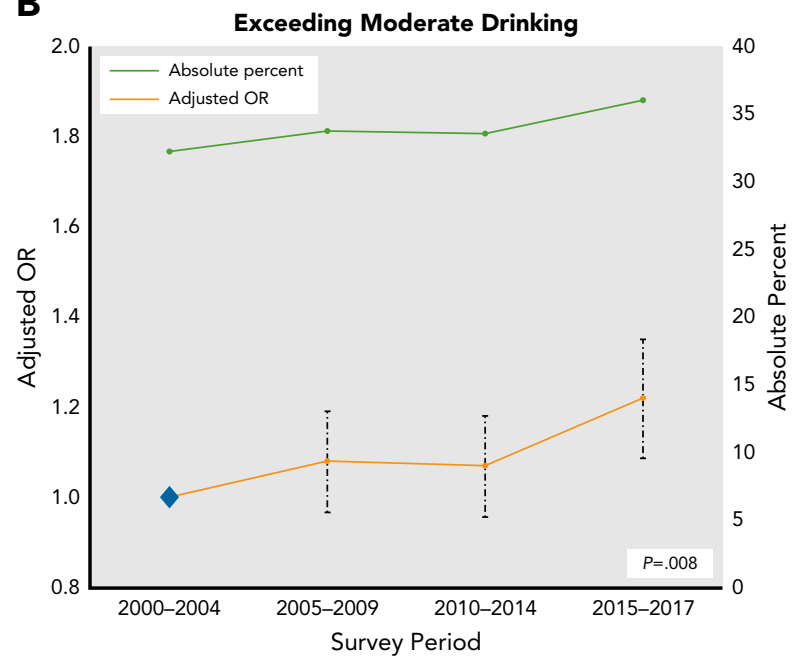

eFigure 1. Adjusted ORs and absolute percents for study participants self-reporting (A) current drinking, (B) exceeding moderate drinking, and (C) binge drinking, by survey period (ref, 2000-2014).

Abbreviation: OR, odds ratio. 


\section{eTable 1. Multivariable Analysis of Participants Reporting a Cancer Diagnosis $>5$ Years Before Survey} Administration $(n=20,828)$

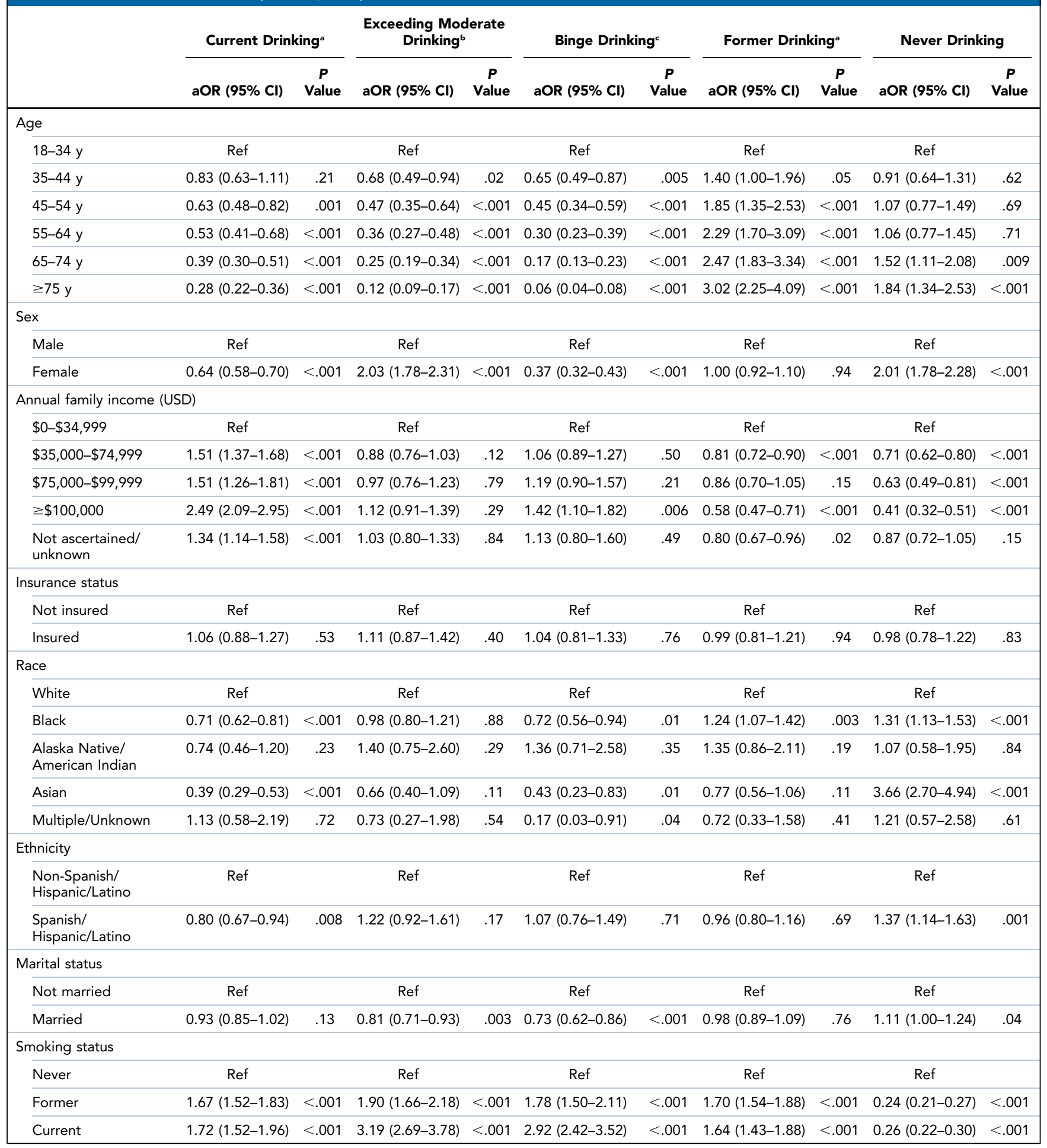




\begin{tabular}{|c|c|c|c|c|c|c|c|c|c|c|}
\hline & \multicolumn{2}{|c|}{ Current Drinkinga } & \multicolumn{2}{|c|}{$\begin{array}{c}\text { Exceeding Moderate } \\
\text { Drinking }^{\mathrm{b}}\end{array}$} & \multicolumn{2}{|c|}{ Binge Drinking $^{c}$} & \multicolumn{2}{|c|}{ Former Drinkinga } & \multicolumn{2}{|c|}{ Never Drinking } \\
\hline & aOR $(95 \% \mathrm{Cl})$ & $\begin{array}{c}P \\
\text { Value }\end{array}$ & aOR $(95 \% \mathrm{Cl})$ & $\begin{array}{c}P \\
\text { Value }\end{array}$ & aOR $(95 \% \mathrm{Cl})$ & $\begin{array}{c}P \\
\text { Value }\end{array}$ & aOR $(95 \% \mathrm{Cl})$ & $\begin{array}{c}P \\
\text { Value }\end{array}$ & aOR $(95 \% \mathrm{Cl})$ & $\begin{array}{c}P \\
\text { Value }\end{array}$ \\
\hline \multicolumn{11}{|l|}{ Education } \\
\hline Grade 5-12 & $2.30(1.34-3.93)$ & .002 & $1.38(0.63-3.03)$ & .42 & $2.43(0.64-9.15)$ & .19 & $0.91(0.58-1.43)$ & .69 & $0.65(0.40-1.06)$ & .09 \\
\hline $1-4$ y college & $3.71(2.16-6.36)$ & $<.001$ & $1.10(0.50-2.41)$ & .82 & $2.12(0.56-7.99)$ & .27 & $0.72(0.46-1.14)$ & .16 & $0.41(0.25-0.67)$ & $<.001$ \\
\hline$\geq 5$ y college & $4.18(2.40-7.28)$ & $<.001$ & $0.85(0.38-1.90)$ & .70 & $1.54(0.40-5.91)$ & .53 & $0.65(0.40-1.05)$ & .08 & $0.39(0.23-0.65)$ & $<.001$ \\
\hline Unknown & $0.94(0.37-2.35)$ & .89 & $1.27(0.27-6.01)$ & .76 & $2.69(0.26-26.40)$ & .40 & $1.57(0.72-3.44)$ & .26 & $0.84(0.40-1.76)$ & .64 \\
\hline Fair & $0.37(0.32-0.43)$ & $<.001$ & $0.71(0.57-0.88)$ & .002 & $0.82(0.64-1.06)$ & .14 & $2.36(1.98-2.81)$ & $<.001$ & $1.64(1.36-1.98)$ & $<.001$ \\
\hline Poor & $0.27(0.22-0.32)$ & $<.001$ & $0.70(0.51-0.96)$ & .03 & $0.59(0.40-0.86)$ & .006 & $2.84(2.31-3.51)$ & $<.001$ & $2.02(1.61-2.52)$ & $<.001$ \\
\hline \multicolumn{11}{|l|}{ Survey period } \\
\hline 2000-2004 & Ref & & Ref & & Ref & & Ref & & Ref & \\
\hline 2005-2009 & $1.00(0.87-1.13)$ & .92 & $0.96(0.79-1.18)$ & .73 & $1.30(1.02-1.66)$ & .03 & $1.10(0.86-1.27)$ & .18 & $0.97(0.84-1.12)$ & .67 \\
\hline 2010-2014 & $1.07(0.94-1.21)$ & .29 & $0.94(0.78-1.13)$ & .52 & $1.34(1.06-1.68)$ & .01 & $1.15(1.00-1.31)$ & .04 & $0.82(0.72-0.94)$ & .004 \\
\hline 2015-2017 & $1.22(1.07-1.41)$ & .003 & $1.00(0.82-1.23)$ & .99 & $1.82(1.42-2.32)$ & $<.001$ & $1.02(0.87-1.18)$ & .84 & $0.79(0.68-0.92)$ & .002 \\
\hline
\end{tabular}

Abbreviation: aOR, adjusted odds ratio.

aAmong all survey participants.

bAmong current drinkers. Defined as $>1$ drink/day for women and $>2$ drinks/day for men.

cAmong current drinkers. Defined as $\geq 5$ drinks/day during at least 1 day over the past year.

\section{eTable 2. Multivariable Analysis by Era of Survey Among Participants Aged $\leq 50$ Years $(n=6,313)$}

\begin{tabular}{|c|c|c|c|c|c|c|}
\hline \multirow[b]{2}{*}{ Survey Period } & \multicolumn{2}{|c|}{ Current Drinking } & \multicolumn{2}{|c|}{ Exceeding Moderate Drinking } & \multicolumn{2}{|c|}{ Binge Drinking } \\
\hline & aOR $(95 \% \mathrm{Cl})$ & $P$ Value & aOR $(95 \% \mathrm{CI})$ & $P$ Value & aOR $(95 \% \mathrm{Cl})$ & $P$ Value \\
\hline 2000-2004 & Ref & & Ref & & Ref & \\
\hline 2005-2009 & $1.12(0.88-1.36)$ & .24 & $0.96(0.75-1.22)$ & .74 & $1.02(0.81-1.29)$ & .87 \\
\hline 2010-2014 & $1.20(0.99-1.46)$ & .07 & $0.86(0.68-1.10)$ & .23 & $1.14(0.90-1.46)$ & .28 \\
\hline 2015-2017 & $1.07(0.85-1.36)$ & .56 & $0.97(0.73-1.27)$ & .81 & $1.48(1.12-1.95)$ & .006 \\
\hline
\end{tabular}

The model was also adjusted for sex, annual family income, race, ethnicity, marital status, smoking status, highest education level, insurance status, and self-reported health status.

Abbreviation: aOR, adjusted odds ratio. 
eTable 3. Multivariable Analysis by Cancer Type of Participants Reporting a Cancer Diagnosis $\geq 5$ Years Before Survey Administration $(n=20,828)$

\begin{tabular}{|c|c|c|c|c|c|c|c|}
\hline Cancer Type & $\mathbf{n}$ & \multicolumn{2}{|c|}{ Current Drinking ${ }^{a}$} & \multicolumn{2}{|c|}{ Exceeding Moderate Drinking } & \multicolumn{2}{|c|}{ Binge Drinking ${ }^{c}$} \\
\hline Breast & 5,394 & $0.76(0.71-0.83)$ & $<.001$ & $0.61(0.54-0.69)$ & $<.001$ & $0.47(0.40-0.55)$ & $<.001$ \\
\hline Prostate & 2,902 & $1.11(1.00-1.22)$ & .04 & $0.56(0.47-0.68)$ & $<.001$ & $0.77(0.65-0.92)$ & .005 \\
\hline Cervical & 2,522 & $1.43(1.28-1.59)$ & $<.001$ & $2.22(1.92-2.66)$ & $<.001$ & $1.58(0.65-0.92)$ & $<.001$ \\
\hline Colorectal & 1,835 & $0.67(0.60-0.76)$ & $<.001$ & $0.74(0.61-0.90)$ & .002 & $1.05(0.84-1.31)$ & .67 \\
\hline Uterine & 1,659 & $0.71(0.63-0.81)$ & $<.001$ & $0.85(0.70-1.03)$ & .09 & $0.71(0.55-0.91)$ & .008 \\
\hline Lung & 546 & $0.74(0.60-0.91)$ & .004 & $0.76(0.54-1.07)$ & .12 & $1.02(0.67-1.56)$ & .92 \\
\hline Bladder & 614 & $1.04(0.85-1.27)$ & .69 & $0.65(0.48-0.90)$ & .008 & $1.17(0.83-1.64)$ & .38 \\
\hline Head and neck & 454 & $1.00(0.78-1.27)$ & .99 & $0.93(0.67-1.30)$ & .69 & $1.49(1.03-2.15)$ & .03 \\
\hline CNS & 181 & $0.81(0.57-1.16)$ & .25 & $1.26(0.76-2.11)$ & .37 & $1.07(0.56-2.04)$ & .85 \\
\hline
\end{tabular}

Abbreviation: aOR, adjusted odds ratio; CNS, central nervous system.

among all survey participants.

${ }^{b}$ Among current drinkers. Defined as $>1$ drink/day for women and $>2$ drinks/day for men.

cAmong current drinkers. Defined as $\geq 5$ drinks/day during at least 1 day over the past year. 